\title{
The Field Generated by an Arbitrary Current Distribution Within a Waveguide
}

\author{
By J. J. Freeman
}

\begin{abstract}
Formulas are derived for the electromagnetic field generated by an arbitrary current distribution within waveguides of rectangular, circular, and coaxial cross sections. These formulas are obtained by generalizing a nalogous formulas obtained in a previous paper ${ }^{2}$ for cavities of the same cross section. As a check on the formulas, it is shown that the field from an axially directed dipole within a circular guide reduces to the free space value as the radius increases indefinitely.
\end{abstract}

\section{Introduction}

In pursuing certain investigations, it was found necessary to derive explicit expressions for the electromagnetic field generated by an arbitrary current distribution within a waveguide. Since the corresponding problem for a cylindrical cavity had already been worked out in a previous paper (see footnote 2), henceforth designated as Paper I, the results of Paper I were generalized to apply to a waveguide of corresponding cross section by the simple expedient of allowing the length of the cavity to become infinitely large. Although the same formulas may be obtained more directly by a different method, ${ }^{3}$ the present paper derives these formulas using the above mentioned limiting process since: (a) no explicit general solution of the problem has yet been published; (b) although the expedient of first using a finite cavity for various computations involving electromagnetic fields, and then allowing the dimensions of the cavity to become infinitely large has been widely employed, the validity of the limiting process is not entirely obvious, and the following derivation throws light on this process.

To avoid repetition it will be assumed that

1 This paper is a revision of Report CRPL-9-3, issued under the same title (Feb. 28, 1947).

2 J. J Freeman, Theory and design of a cavity attenuator, J. Research NBS 40, 235 (1948) RP1868.

3 J. J. Freeman, The noise spectrum of a diode with a retarding field, J. Research NBS 42, 75 (1949) RP 1953 (see appendix I).
Paper $I$ is available to the reader, and the results contained therein will be utilized without further comment. MKS units are employed. Although it was assumed in Paper I that the medium within the cavity was lossless, and that the wave number, $k=\omega \sqrt{\mu \epsilon}$, was real, the results are still valid for complex values of $k$. Accordingly, in the following, we assume that the medium has conductivity $\sigma$, so that

$$
k^{2}=\omega^{2} \mu \epsilon+j \mu \omega \sigma
$$

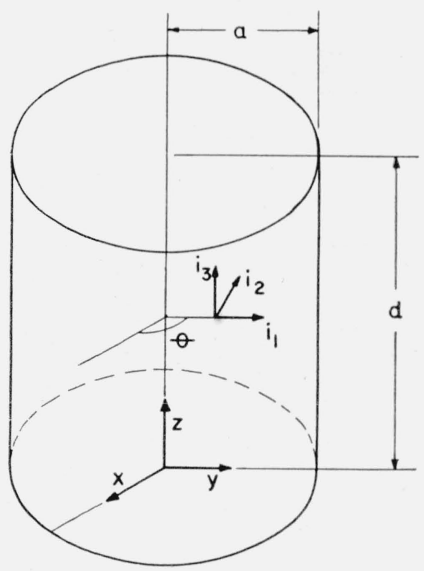

Figure 1. Cylindrical cavity.

\section{The Circular Cylindrical Waveguide}

Consider a cylindrical cavity, of length $d$ (fig. 1) containing current of volume density $\boldsymbol{J}\left(\boldsymbol{r}^{\prime}\right)$, where $\boldsymbol{r}^{\prime}$ designates the position vector of $\boldsymbol{J}$, and 
where the time variation, $e^{j \omega t}$ is omitted. In eq 15 and 21 , Paper I, it was shown that

$$
\begin{aligned}
& \boldsymbol{E}_{T E}=j \omega \mu \sum_{n=0}^{\infty} \sum_{m=1}^{\infty} A_{n m}^{2} \int \boldsymbol{J}\left(\boldsymbol{r}^{\prime}\right) \cdot \boldsymbol{m}\left(\boldsymbol{r}^{\prime}\right) \sum_{l=1}^{\infty} \\
& \frac{\sin \frac{l \pi z^{\prime}}{d} \sin \frac{l \pi z}{d}}{\delta^{2}-k^{2}+\frac{l^{2} \pi^{2}}{d^{2}}} d \tau^{\prime},
\end{aligned}
$$

where $l, m, n,=0,1,2, \ldots$ and,

$$
\begin{aligned}
& \boldsymbol{E}_{T M}=\frac{j}{\omega \epsilon} \int \boldsymbol{J}\left(\boldsymbol{r}^{\prime}\right) \cdot \sum_{n=0}^{\infty} \sum_{m=1}^{\infty} F_{n m}^{2}\left\{\boldsymbol{n}\left(\boldsymbol{r}^{\prime}\right) \boldsymbol{n}(\boldsymbol{r})\right. \\
& \frac{k^{2}-\lambda^{2}}{\lambda^{2}} \sum_{l=1}^{\infty} \frac{\sin \frac{l \pi z^{\prime}}{d} \sin \frac{l \pi z}{d}}{\lambda^{2}-k^{2}+\frac{l^{2} \pi^{2}}{d^{2}}}+\boldsymbol{n}\left(\boldsymbol{r}^{\prime}\right) \boldsymbol{p}(\boldsymbol{r}) \\
& \sum_{l=1}^{\infty} \frac{\frac{l \pi}{d} \sin \frac{l \pi z^{\prime}}{d} \cos \frac{l \pi z}{d}}{\lambda^{2}-k^{2}+\frac{l^{2} \pi^{2}}{d^{2}}}+\boldsymbol{p}\left(\boldsymbol{r}^{\prime}\right) \boldsymbol{n}(\boldsymbol{r}) \sum_{l=1}^{\infty} \\
& \frac{\frac{l \pi}{d} \cos \frac{l \pi z^{\prime}}{d} \sin \frac{l \pi z}{d}}{\lambda^{2}-k^{2}+\frac{l^{2} \pi^{2}}{d^{2}}}+\boldsymbol{p}\left(\boldsymbol{r}^{\prime}\right) \boldsymbol{p}(\boldsymbol{r}) \sum_{l=0}^{\infty} \\
& \left.\frac{\left.\left(k^{2}-\frac{l^{2} \pi^{2}}{d^{2}}\right) \cos \frac{l \pi z^{\prime}}{d} \cos \frac{l \pi z}{d}\right)}{\left(\lambda^{2}-k^{2}+\frac{l^{2} \pi^{2}}{d^{2}}\right)\left(1+\delta_{0}^{l}\right)}\right\} d \tau^{\prime} \\
& \delta_{0}^{l}=\begin{array}{l}
0, \text { for } l \neq 00 \\
1, \text { for } l=1
\end{array}
\end{aligned}
$$

In the above, $d \tau^{\prime}$ represents an element of volume containing $\boldsymbol{J}$, and $\boldsymbol{r}$ is the position vector of the point of observation. The $\boldsymbol{m}, \boldsymbol{n}, \boldsymbol{p}, \delta$, and $\lambda$ in eq 1 and 2 have the identifying subscripts $m n$ implied but omitted. Also, the even, odd designations $\left(\begin{array}{l}e \\ o\end{array}\right)$ are omitted for convenience, but are implied. For the case of a cavity of circular cross-section, of radius $a$, (fig. 1),

$$
\underset{n m}{\boldsymbol{m}_{\mathrm{o}}^{\mathrm{e}}}=\frac{n}{r}\left(\begin{array}{r}
-\sin n \theta \\
\cos n \theta
\end{array}\right) J_{n}(\delta r) \boldsymbol{i}_{1}-\left(\begin{array}{c}
\cos n \theta \\
\sin n \theta
\end{array}\right) \delta J_{\mathrm{n}}^{\prime}(\delta r) \boldsymbol{i}_{2},
$$

where $\boldsymbol{i}_{1}, \boldsymbol{i}_{2}$, and $\boldsymbol{i}_{3}$ are unit vectors in the directions of increasing $r, \theta$, and $z$, and

$$
\begin{array}{cl}
A_{n m}^{e}=\frac{\sqrt{2 \beta_{n}}}{\sqrt{\pi d} \sqrt{v_{n m}^{2}-n^{2} J_{n}\left(v_{n m}\right)}} & \beta_{n}=1 \text { for } n=0 \\
\beta_{n}=2 \text { for } n \neq 0 .
\end{array}
$$

$$
\delta=\delta_{n m}=\frac{v_{n m}}{a} \text {, where } J_{n}^{\prime}\left(v_{n m}\right)=0 .
$$

Also,

$$
\boldsymbol{n}_{o}^{e}=-\lambda J_{n}^{\prime}(\lambda r)\left(\begin{array}{c}
\cos n \theta \\
\sin n \theta
\end{array}\right) \boldsymbol{i}_{1}+\left(\begin{array}{r}
\sin n \theta \\
-\cos n \theta
\end{array}\right) \frac{n}{r} J_{n}(\delta r) \boldsymbol{i}_{2},
$$

$$
\underset{n m}{\boldsymbol{p}_{n}^{e}}=\left(\begin{array}{c}
\cos n \theta \\
\sin n \theta
\end{array}\right) J_{n}(\lambda r) \boldsymbol{i}_{3},
$$

$$
\begin{gathered}
F_{n m}=\frac{\sqrt{2 \beta_{n}}}{a \sqrt{\pi d} J_{n-1}(\lambda a)}, \\
\lambda=\lambda_{n m}=\frac{u_{n m}}{a} \text {, where } J_{n}\left(u_{n m}\right)=0 .
\end{gathered}
$$

To generalize eq 1 and 2 to the case of an infinite waveguide, with a conducting plug at $z=0$, we let $d \rightarrow \infty$, and evaluate the limit of the infinite summation over the index $l$ as $d \rightarrow \infty$.

Consider the sum over $l$ in eq 1, multiplied by the factor $1 / d$.

$S_{1}=\frac{1}{d} \sum_{l=1}^{\infty} \frac{\sin \frac{l \pi z^{\prime}}{d} \sin \frac{l \pi z}{d}}{\frac{l^{2} \pi^{2}}{d^{2}}-g^{2}}=1 / 2 d \sum_{l=-\infty}^{\infty} \frac{\sin \frac{l \pi z^{\prime}}{d} \sin \frac{l \pi z}{d}}{\frac{l^{2} \pi^{2}}{d^{2}}-g^{2}}$,

where

$$
g^{2}=k^{2}-\delta^{2} .
$$

The factor $1 / d$ enters because it is contained in the normalizing factor $A_{n m}^{2}$, (eq 4). Let $\xi=l \pi / d$. Then

$S_{1}=1 / 2 d \sum_{l=-\infty}^{\infty} \frac{\sin \xi z^{\prime} \sin \xi z}{\xi^{2}-g^{2}} \Delta l=\frac{1}{2 \pi} \sum_{\xi} \frac{\sin \xi z^{\prime} \sin \xi z}{\xi^{2}-g^{2}} \Delta \xi$,

since $\Delta l=1$. As $d \rightarrow \infty, \Delta \xi \rightarrow 0$, and the sum becomes in the limit the integral,

$$
\begin{aligned}
S_{1}= & \frac{1}{2 \pi} \int_{-\infty}^{\infty} \frac{\sin \xi z^{\prime} \sin \xi z d \xi}{\xi^{2}-g^{2}}= \\
& \frac{1}{4 \pi} \int_{-\infty}^{\infty} \frac{\cos \xi\left(z-z^{\prime}\right)-\cos \xi\left(z+z^{\prime}\right)}{\xi^{2}-g^{2}} d \xi .
\end{aligned}
$$

Since

$\int_{-\infty}^{\infty} \frac{\sin \xi\left(z-z^{\prime}\right) d \xi}{\xi^{2}-g^{2}}=0$, because the sin is an odd function,

$$
S_{1}=\frac{1}{4 \pi} \int_{-\infty}^{\infty} \frac{e^{j \xi\left(z-z^{\prime}\right)}-e^{j \xi\left(z+z^{\prime}\right)}}{\xi^{2}-g^{2}} d \xi .
$$

Let $g_{1}$ and $g_{2}$ be the two square roots of $g^{2}=k^{2}-\delta^{2}$, 
which lie in the positive- and negative-imaginary parts of the complex plane, respectively. Then

$$
g_{2}=-g_{1} .
$$

For frequencies above cut-off, when $\omega^{2} \mu \epsilon>\delta^{2}$,

where

$$
g_{1}=|g| e \frac{j \psi}{2}
$$

$$
|g|=\left[\left(\omega^{2} \mu \epsilon-\delta^{2}\right)^{2}+\omega^{2} \mu^{2} \sigma^{2}\right]^{\frac{1}{2}}
$$

and

$$
\tan \psi=\frac{\omega \mu \sigma}{\left|\omega^{2} \mu \epsilon-\delta^{2}\right|} .
$$

For frequencies below cut-off, when $\omega^{2} \mu \epsilon<\delta^{2}$,

$$
g_{1}=j|g| e \frac{-j \psi}{2} .
$$

Consider

$$
\int_{-\infty}^{\infty} \frac{e^{j \xi\left(2-z^{\prime}\right)} d \xi}{\xi^{2}-g^{2}}
$$

Since, for $z>z^{\prime}, \frac{e^{j \xi\left(z-z^{\prime}\right)}}{\xi^{2}-g^{2}}$

vanishes over the positive-imaginary part of the plane, as $|\xi| \rightarrow \infty$, the integral can be evaluated by taking the residue around the pole $g_{1}$ (fig. 2). Thus,

$$
\int_{-\infty}^{\infty} \frac{e^{j \xi\left(z-z^{\prime}\right)}}{(\xi+g)(\xi-g)} d \xi=\frac{2 \pi j e^{j g_{1}\left(z-z^{\prime}\right)}}{2 g_{1}} .
$$

For $z<z^{\prime}, \frac{e^{j \xi\left(z-z^{\prime}\right)}}{\xi^{2}-g^{2}}$ vanishes over the negativeimaginary half-plane, as $|\xi| \rightarrow \infty$, and the integral can be evaluated by taking the residue around the pole (fig. 2) $g_{2}$. Thus, for $z<z^{\prime}$,

$$
\int_{-\infty}^{\infty} \frac{e^{j \xi\left(z-z^{\prime}\right)}}{\xi^{2}-g^{2}} d \xi=-2 \pi j \frac{e^{j g_{2}\left(z-z^{\prime}\right)}}{2 g_{2}}=\frac{2 \pi j e^{-j g_{1}\left(z-z^{\prime}\right)}}{2 g_{1}} .
$$

or

$$
\int_{-\infty}^{\infty} \frac{e^{j \xi\left(z-z^{\prime}\right)}}{\xi^{2}-\overline{g^{2}}} d \xi=\frac{\pi j}{g_{1}} e^{j g_{1}\left|z-z^{\prime}\right|} \text { for } z \gtrless z^{\prime}
$$

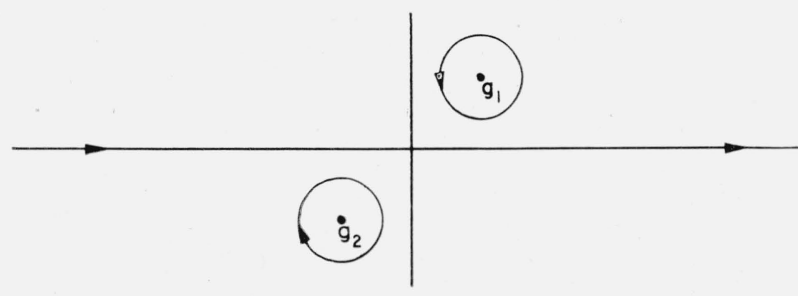

Figure 2. Contour of integration path in complex plane.

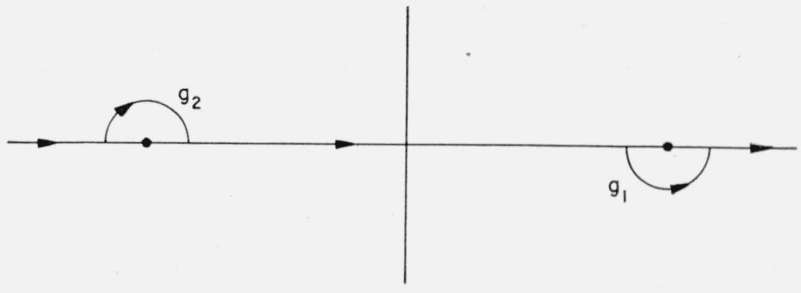

Figure 3. Contour of integration path used by Sommerfeld.

If $\sigma=0$, instead of integrating along the real axis of the $s$-plane it becomes necessary to indent the path at $g_{1}$ and $g_{2}$ as shown in figure 3 , obtaining the contour that Sommerfeld ${ }^{4}$ used in the complex integral representation of the Green's function for the one-dimensional wave equation.

From eq 11, one recognizes that $S_{1}$ represents two plane waves traveling in the $+z$-direction (for $z>z^{\prime}$ ), one having its source at $z=z^{\prime}$, and the other at $z=-z^{\prime}$. Also, since $\boldsymbol{m}$ is a vector lying in the plane perpendicular to the $z$-direction and since only transverse elements of current make a contribution to the field in eq 1, the first integral in eq 11 represents the contribution due to a current element at $z=z^{\prime}$, and the second integral represents the contribution of its electrical image, situated at $z=-z^{\prime}$. Both element and image are necessary to insure the vanishing of the tangential component of $\boldsymbol{E}$ at $z=0$, which corresponds to the case of a conducting plug at $z=0$. On the other hand, if the guide is infinitely long in the negative $z$-direction, then the contribution of the image at $z=-z^{\prime}$ must be omitted, and the second integral in eq 11 must be neglected.

Since the case of a perfectly conducting plug at $z=0$ may be easily derived from the case of an infinite guide by superposing the electrical image with respect to the plane $z=0$, the following is restricted to the case of a guide extending to infinity in both directions.

Accordingly, from eq 11 and 17, neglecting the contribution from the image,

$$
S_{1}=\frac{j}{4 g_{1}} e^{j g_{1}\left|z-z^{\prime}\right|}\left(z \gtrless z^{\prime}\right) .
$$

Substituting eq 18 in eq 1 ,

$$
\begin{gathered}
E_{T E}=\frac{-\omega \mu}{2 \pi} \sum_{n=0}^{\infty} \sum_{m=1}^{\infty} \frac{\beta_{n} \boldsymbol{J} \boldsymbol{J} \cdot \boldsymbol{m}\left(\boldsymbol{r}^{\prime}\right) e^{j g_{1} \mid z-z^{\prime \prime}} \boldsymbol{m}(\boldsymbol{r})}{g_{1}\left(v_{n m}^{2}-n^{2}\right) \boldsymbol{J}_{n}^{2}\left(v_{n m}\right)} d \tau^{\prime} \\
\left(z \gtrless z^{\prime}\right) .
\end{gathered}
$$

${ }^{4}$ A. Sommerfeld, Deutsche Mathematiker-Vereinigung Jahresbericht, 21, 309 (1912). 
The various summations over the index $l$ in eq 2 may likewise be extended to the case of a guide extending to infinity in the positive $z$-direction, with a conducting plug at $z=0$. By omitting contributions to the field arising from the electrical image with respect to the perfectly conducting plane at $z=0$ of current elements within the guide, the boundary conditions are changed from the case of a perfectly conducting plug to the case of a guide infinite in both directions. Then, if one computes the limiting values of the summations by the method of residues and omits the contribution from the image one obtains:

$\frac{1}{d} \sum_{l=0}^{\infty} \frac{\cos \frac{l \pi z^{\prime}}{d} \cos \frac{l \pi z}{d}}{\left(1+\delta_{0}^{l}\right)\left(\frac{l^{2} \pi^{2}}{d^{2}}-h^{2}\right)}=\frac{j}{4 h_{1}} e^{j h_{1}\left|z-z^{\prime}\right|}\left(z \gtrless z^{\prime}\right)$

$\frac{1}{d} \sum_{l=0}^{\infty} \frac{\frac{l^{2} \pi^{2}}{d^{2}} \cos \frac{l \pi z^{\prime}}{d} \cos \frac{l \pi z}{d}}{\frac{l^{2} \pi^{2}}{d^{2}}-h^{2}}=\frac{j h_{1}}{4} e^{+j h_{1}\left|z-z^{\prime}\right|}\left(z \gtrless z^{\prime}\right)$

$\frac{1}{d} \sum_{l=0}^{\infty} \frac{\frac{l \pi}{d} \sin \frac{l \pi z^{\prime}}{d}}{\frac{l^{2} \pi^{2}}{d^{2}}-h^{2}} \cos \frac{l \pi z}{d}=\mp \frac{1}{4} e^{+j h|| z-z^{\prime} \mid}\left(z \gtrless z^{\prime}\right)$

$$
\frac{1}{d} \sum_{l=0}^{\infty} \frac{\frac{l \pi}{d} \cos \frac{l \pi z^{\prime}}{d} \sin \frac{l \pi z}{d}}{\frac{l^{2} \pi^{2}}{d^{2}}-h^{2}}= \pm \frac{1}{4} e^{+j h_{1}\left|z-z^{\prime}\right|}\left(z \gtrless z^{\prime}\right),
$$

where, for brevity,

$$
h^{2}=k^{2}-\lambda^{2} .
$$

If eq $8,18,20,21,22$, and 23 , are substituted in eq 2 , one finally obtains

$$
\begin{aligned}
E_{T M}= & -\frac{\boldsymbol{S} \boldsymbol{J}\left(\boldsymbol{r}^{\prime}\right)}{2 \pi \omega \epsilon a^{2}} \cdot\left\{\sum_{n=0}^{\infty} \sum_{m=1}^{\infty} \frac{\beta_{n}}{J_{n-1}^{2}\left(u_{n m}\right)}\right. \\
& {\left[\frac{h_{1} a^{2}}{u_{n m}^{2}} \boldsymbol{n}\left(\boldsymbol{r}^{\prime}\right) \boldsymbol{n}(\boldsymbol{r}) \pm j \boldsymbol{n}\left(\boldsymbol{r}^{\prime}\right) \boldsymbol{p}(\boldsymbol{r}) \mp j \boldsymbol{p}\left(\boldsymbol{r}^{\prime}\right) \boldsymbol{n}(\boldsymbol{r})+\right.} \\
& \left.\left.\frac{u_{n m}^{2}}{h_{1} a^{2}} \boldsymbol{p}\left(\boldsymbol{r}^{\prime}\right) \boldsymbol{p}(\boldsymbol{r})\right] e^{+j h_{1}\left|z-z^{\prime}\right|}\right\} d \tau^{\prime} \text { for } z \gtrless z^{\prime} . \quad(24)
\end{aligned}
$$

Here $h_{1}$ is that square root of $k^{2}-\lambda^{2}$ that lies in the positive-imaginary half-plane.

As a check on the derivation, it will be shown that waveguide eq 19 and 24 reduce to the correct value for the free space field, as the boundaries recede indefinitely.

Consider a current filament of length $d l$, carrying current $I$ parallel to the axis of the guide and situated at the origin of coordinates. Then $E_{T E}=0$, and eq 24 becomes,

$$
E_{T M}=\frac{-1}{2 \pi \omega \epsilon a^{2}} \sum_{m=1}^{\infty} \frac{\boldsymbol{S} \boldsymbol{J} \cdot \boldsymbol{p} d \tau^{\prime}}{J_{1}^{2}\left(u_{o m}\right)}\left[-j \boldsymbol{n} \cdot+\frac{u_{o m}^{2} \boldsymbol{p}}{h_{1} a^{2}}\right] e^{j h_{1} z},
$$

since only the symmetric modes for which $n=0$ are excited, and $J$ is normal to $\boldsymbol{n}$.

$$
\int \boldsymbol{J} \cdot \boldsymbol{p} d \tau^{\prime}=\int \boldsymbol{J} \cdot \boldsymbol{i}_{3} J_{0}\left(\lambda r^{\prime}\right) d \tau^{\prime}=I d l .
$$

Since $\boldsymbol{n}_{o m}=-\lambda J_{o}^{\prime}(\lambda r) \boldsymbol{i}_{1}=-\frac{d}{d r} J_{o}(\lambda r) i_{1}$, from eq 6 , and $\boldsymbol{p}_{\text {om }}=J_{o}(\lambda r) \boldsymbol{i}_{3}$, from eq 7 , eq 25 becomes

$$
\begin{aligned}
& E_{T \cdot M}=\frac{-I d l}{2 \pi \omega \epsilon a^{2}} \sum_{m=1}^{\infty} \\
& {\left[\frac{j \frac{d}{d r}\left(J_{o}(\lambda r)\right) \boldsymbol{i}_{1}+{ }_{h_{1} a^{2}}^{u_{o}^{2}} J_{o}(\lambda r) \boldsymbol{i}_{3}}{J_{1}^{2}\left(u_{o m}\right)}\right] e^{j h_{1} z} .}
\end{aligned}
$$

Equation 26 may be rearranged into the following more convenient form:

$E_{T M M}=\frac{-I d l}{2 \pi \epsilon \omega}\left(\operatorname{grad} \operatorname{div}+k^{2}\right) \sum_{m=1}^{\infty} \frac{J_{o}(\lambda r) e^{j h_{1} z}}{h_{1} a^{2} J_{1}^{2}\left(u_{o m}\right)} \boldsymbol{i}_{3}$.

Consider the sum

$$
S=\sum_{m=1}^{\infty} \frac{J_{o}(\lambda r) e^{j h_{1} z}}{h_{1} a^{2} J_{1}^{2}\left(u_{o m}\right)} .
$$

There exists an integer $M$ such that for $m>M$, $u_{o m}$ will be large enough so that $J_{0}\left(u_{o m}\right)$ may be replaced by its asymptotic value ${ }^{5}$,

$$
J_{0}\left(u_{o m}\right) \simeq \sqrt{\frac{2}{\pi u_{o m}}} \cos \left(u_{o m}-\frac{2 n+1}{4} \pi\right) .
$$

Since $u_{o m}$ is the $m^{\text {th }}$ root of $J_{o}\left(u_{o m}\right)=0$, eq 29 yields, for $m>M$,

$$
u_{o m}=\pi(m+3 / 4) .
$$

Accordingly,

$$
J_{1}^{2}\left(u_{o m}\right)=2 / \pi u_{o m}, m>M .
$$

Equation 28 may then be separated into two sums,

\footnotetext{
${ }^{5}$ J. A. Stratton, Electromagnetic theory, p. 359, eq 18 (McGraw-Hill Book Co., Inc., New York, N. Y., 1941).
} 


$$
S=\sum_{m=1}^{M} \frac{J_{o}(\lambda r) e^{j h_{1} z}}{h_{1} a^{2} J_{1}^{2}\left(u_{o m}\right)}+\frac{1}{2} \sum_{m=M}^{\infty} \frac{\pi u_{o m} J_{o}(\lambda r) e^{j h_{1} z}}{a^{2} h_{1}} \Delta m
$$

From eq $30, \Delta m=\frac{\Delta u_{o m}}{\pi}$, and since $\lambda=\frac{u_{o m}}{a}$,

$$
\Delta m=\frac{a}{\pi} \Delta \lambda .
$$

Accordingly, eq 32 may be rewritten as follows:

$$
S=\sum_{m=1}^{M} \frac{J_{o}(\lambda r) e^{j h_{1} z}}{h_{1} a^{2} J_{1}^{2}\left(u_{o m}\right)}+\frac{1}{2} \sum_{\lambda=\frac{\pi}{a}}^{\infty} \frac{J_{o}(\lambda r) \lambda e^{j h_{1} z}}{h_{1}} \Delta \lambda .
$$

Now let $a \rightarrow \infty$. As $a$ increases without limit the first sum in eq 34 approaches zero, $\Delta \lambda \rightarrow 0$; the second summation becomes, by definition, an integral; and the lower limit of integration becomes zero.

Thus, as $a \rightarrow \infty$,

$$
S=\frac{1}{2} \int_{0}^{\infty} \frac{J_{o}(\lambda r) e^{j h_{1} z} \lambda}{h_{1}} d \lambda .
$$

Substituting from (eq 23a),

$$
j h_{1}=-\sqrt{\lambda^{2}-k^{2}}
$$

into eq 35 , one obtains

$$
S=\frac{1}{2 j} \int_{0}^{\infty} \frac{J_{o}(\lambda r) e^{-\sqrt{\lambda^{2}-k^{2}} z} \lambda}{\left(\lambda^{2}-k^{2}\right)^{1 / 2}} d \lambda
$$

where that square root is to be taken whose real part is positive.

But

$$
\int_{0}^{\infty} \frac{J_{0}(\lambda r) e^{-\sqrt{\lambda^{2}-k^{2}} z}}{\left(\lambda^{2}-k^{2}\right)^{1 / 2}} \lambda d \lambda
$$

is the well-known ${ }^{6}$ expansion for $\frac{e^{j k R}}{R}$ where $R=\sqrt{r^{2}+z^{2}}$. Accordingly, eq 27 becomes

$$
E_{T M}=\left(\operatorname{grad} \operatorname{div}+k^{2}\right)\left(\frac{j I d l}{4 \pi \omega \epsilon} \frac{e^{j k R}}{R} \boldsymbol{i}_{3}\right)
$$

which is the field due to a current element $I d l \boldsymbol{i}_{3}$ in free space. ${ }^{7}$

\footnotetext{
${ }^{6}$ J. A. Stratton, Electromagnetic theory, p. 576, eq 17 (McGraw Hill Book Co., Inc., New York, N. Y., 1941).

- J. A. Stratton, Electromagnetic theory, p. 431, eq 44 (McGrawHill Book Co., Inc., New York, N. Y., 1941).
}

\section{Rectangular Cross Section}

The same general formulas (eq 1 and 2), hold in the case of a cavity of length $c$, and of rectangular cross section, whose $x$ and $y$ dimensions are $a$

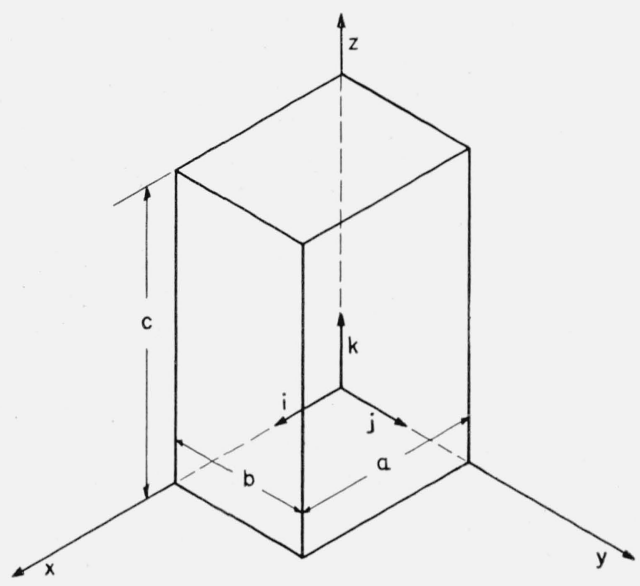

FiguRE 4. Cavity of rectangular cross section.

and $b$, respectively, with the origin at one corner (fig. 4). Here, however, the normalizing factors and vector functions corresponding to the rectangular cross section are as follows:

$$
\begin{gathered}
\boldsymbol{m}_{n m}=\left[\frac{-m \pi}{b} \sin \frac{m \pi y}{b} \cos \frac{n \pi x}{a} \boldsymbol{i}+\right. \\
\left.\frac{n \pi}{a} \sin \frac{n \pi x}{a} \cos \frac{m \pi y}{b} \boldsymbol{j}\right] \\
\boldsymbol{n}_{n m}=-\left[\frac{n \pi}{a} \cos \frac{n \pi x}{a} \sin \frac{m \pi y}{b} \boldsymbol{i}+\right. \\
\left.\frac{m \pi}{b} \cos \frac{m \pi y}{b} \sin \frac{n \pi x}{a} \boldsymbol{j}\right] \\
\boldsymbol{p}_{n m}=\sin \frac{n \pi x}{a} \sin \frac{m \pi y}{b} \boldsymbol{k} \\
A_{n m}=\frac{1}{\lambda_{n m}}\left(\frac{\beta_{n m}}{a b c}\right)^{3 / 2}
\end{gathered}
$$

where

$$
\begin{gathered}
\beta_{n m}=\begin{array}{l}
1 \text { for } n \text { or } m=0 \\
2 \text { for } n \text { and } m \neq 0
\end{array} \\
\lambda_{n m}=\left[\frac{n^{2}}{a^{2}}+\frac{m^{2}}{b^{2}}\right]^{1 / 2} \\
F=\left(\frac{8}{a b c}\right)^{3 / 2} .
\end{gathered}
$$

Accordingly, when eq 38 through eq 44 are substituted in eq 1 and 2, and eq 18, 20 to 23 are 
substituted for the infinite summations over the index $l$, one gets for the case of a guide infinite in both directions,

$$
\begin{aligned}
E_{T E}= & \frac{-\omega \beta}{a b} \sum_{n=0}^{\infty} \sum_{m=0}^{\infty} \frac{\beta_{n m}}{\lambda_{n m}^{2} g} \int \boldsymbol{J}\left(\boldsymbol{r}^{\prime}\right) \cdot \boldsymbol{m}\left(\boldsymbol{r}^{\prime}\right) \\
E_{T M}= & \frac{-1}{\omega \epsilon a b} \int \boldsymbol{J}\left(\boldsymbol{r}^{\prime}\right) \sum_{n=0}^{\infty} \sum_{m=0}^{\infty} \beta_{n m}\left\{\boldsymbol{n}\left(\boldsymbol{r}^{\prime}\right) \boldsymbol{n}(\boldsymbol{r}) \frac{g}{\lambda_{n m}^{2}} \pm\right. \\
& \left.j \boldsymbol{n}\left(\boldsymbol{r}^{\prime}\right) \boldsymbol{p}(\boldsymbol{r}) \mp j \boldsymbol{p}\left(\boldsymbol{r}^{\prime}\right) \boldsymbol{n}(\boldsymbol{r})+\frac{\lambda_{n m}^{2}}{g} \boldsymbol{p}\left(\boldsymbol{r}^{\prime}\right) \boldsymbol{p}(\boldsymbol{r})\right\} \\
& e^{ \pm j g\left(z-z^{\prime}\right)} d \tau^{\prime} \text { for } z \gtrless z^{\prime} .
\end{aligned}
$$

Here, $g$ is that square root of

$$
g^{2}=k^{2}-\frac{n^{2}}{a^{2}}-\frac{m^{2}}{b^{2}},
$$

that lies in the positive-imaginary part of the complex plane.

\section{Coaxial Cross Section}

Similar computations for the case of a coaxial cross-section guide of inner radius $a$ and outer radius $b$ yield the following formulas:

$$
\begin{aligned}
& E_{T E}=\frac{-\omega \mu \pi}{8} \sum_{n=0}^{\infty} \sum_{m=1}^{\infty} \\
& \frac{\beta_{n} J_{n}^{\prime 2}(\eta b) \boldsymbol{S} \boldsymbol{J}\left(\boldsymbol{r}^{\prime}\right) \cdot \boldsymbol{m}\left(\boldsymbol{r}^{\prime}\right) \boldsymbol{e}^{ \pm j g\left(z-z^{\prime}\right)} d \tau^{\prime} \boldsymbol{m}(\boldsymbol{r})}{\left\{J_{n}^{\prime 2}(\eta a)\left(1-\frac{n^{2}}{\eta^{2} b^{2}}\right)-J_{n}^{\prime 2}(\eta b)\left(1-\frac{n^{2}}{\eta^{2} a^{2}}\right)\right\} g} z \gtrless z^{\prime}
\end{aligned}
$$

where

$$
\begin{gathered}
\underset{\boldsymbol{m}_{n \mathrm{~m}}^{e}=}{\boldsymbol{c}^{e}}=\frac{n}{r}\left(\begin{array}{r}
-\sin \mathrm{n} \theta \\
\cos \mathrm{n} \theta
\end{array}\right) \hat{Z}_{n}(\eta r) \boldsymbol{i}_{1}-\left(\begin{array}{c}
\cos \mathrm{n} \theta \\
\sin \mathrm{n} \theta
\end{array}\right) \hat{Z}_{n}^{\prime}(\eta r) \boldsymbol{i}_{2}, \\
\hat{Z}_{n}(\eta r)=J_{n}(\eta r) N_{n}^{\prime}(\eta a)-N_{n}(\eta r) J_{n}^{\prime}(\eta a),
\end{gathered}
$$

and

$$
\eta_{n m} \text { is a root of }\left|\begin{array}{ll}
J_{n}^{\prime}(\eta a) & N_{n}^{\prime}(\eta a) \\
J_{n}^{\prime}(\eta b) & N_{n}^{\prime}(\eta b)
\end{array}\right|=0,
$$

Here, $g$ is that root of

$$
g^{2}=k^{2}-\eta^{2}
$$

that lies in the positive-imaginary part of the plane.

$$
\begin{gathered}
E_{T M}=-\frac{\pi}{8 \omega \boldsymbol{\epsilon}} \sum_{n=0}^{\infty} \sum_{m=1}^{\infty} \frac{\beta_{n} \lambda_{n m}^{2} J_{n}^{2}(\lambda b)}{\boldsymbol{J}_{n}^{2}(\lambda a)-J_{n}^{2}(\lambda b)} \mathcal{J}\left(\boldsymbol{r}^{\prime}\right) . \\
\left\{\frac{h}{\lambda^{2}} n n \pm j \boldsymbol{n p} \mp j \boldsymbol{p} \boldsymbol{n}+\frac{\lambda^{2}}{g} \boldsymbol{p} \boldsymbol{p}\right\} e^{ \pm j h\left(z-z^{\prime}\right)} d \tau^{\prime}+ \\
\frac{\sqrt{\frac{\bar{\mu}}{\epsilon}}}{4 \pi \ln \frac{b}{a}} \mathcal{J} \cdot \frac{\boldsymbol{i}_{1}}{r^{\prime}} \frac{\boldsymbol{i}_{1}}{r} e^{ \pm j k\left(z-z^{\prime}\right)} d \tau^{\prime} z \gtrless z^{\prime} .
\end{gathered}
$$

The unit vectors $i_{1}, i_{2}, i_{3}$ are defined in figure 1 . Here

$$
\begin{gathered}
\underset{\substack{\boldsymbol{n}_{o m}^{e} \\
o}}{e}\left[-\alpha Z_{n}^{\prime}(\alpha r)\left(\begin{array}{c}
\cos n \theta \\
\sin n \theta
\end{array}\right) \boldsymbol{i}_{1}+\left(\begin{array}{c}
\sin n \theta \\
-\cos n \theta
\end{array}\right) \frac{n}{r} Z_{n} \boldsymbol{i}_{2}\right] \\
\boldsymbol{p}_{n m}^{e}=\left(\begin{array}{c}
\cos n \theta \\
\sin n \theta
\end{array}\right) Z_{n}(\alpha r) \boldsymbol{i}_{3}, \\
Z_{n}(\alpha r)=J_{n}(\alpha r) N_{n}(\alpha a)-N_{n}(\alpha r) J_{n}(\alpha a), \\
\text { where } \alpha_{n m} \text { is a solution of }\left|\begin{array}{cc}
J_{n}(\alpha a) & N_{n}(\alpha a) \\
J_{n}(\alpha b) & N_{n}(\alpha b)
\end{array}\right|=0
\end{gathered}
$$

and $h$ is that square root of

$$
h^{2}=k^{2}-\alpha^{2},
$$

which lies in the positive-imaginary part of the plane.

\section{Conclusion}

The fields within a waveguide excited by an arbitrary current distribution have been calculated for the cases of circular, rectangular, and coaxial cross section by taking the limit of the corresponding expressions for cylindrical cavities of corresponding cross section as the length of the cavities becomes infinitely great.

Washington, April 20, 1949. 